PROCEEDINGS OF THE

AMERICAN MATHEMATICAL SOCIETY

Volume 128, Number 5, Pages 1547-1554

S 0002-9939(99)05132-1

Article electronically published on October 5, 1999

\title{
STRICT REGULAR COMPLETIONS OF CAUCHY SPACES
}

\author{
PAUL BROCK AND GARY RICHARDSON
}

(Communicated by Alan Dow)

\begin{abstract}
A diagonal condition is defined and used in characterizing the Cauchy spaces which have a strict, regular completion.
\end{abstract}

\section{INTRODUCTION}

Cauchy spaces were introduced in order to study completions from a general point of view. The first study of completions resembling Cauchy spaces defined here seems to be due to Kowalsky [13]. Keller 9] gave the present definition of Cauchy spaces by characterizing the permissible set of Cauchy filters for a uniform convergence space introduced by Cook and Fischer 4. Early efforts to develop a completion theory for uniform convergence spaces led to the realization that Cauchy spaces provide a more natural foundation, and subsequent research shifted to Cauchy spaces. Cauchy spaces have been used in diverse areas; for example, Ball [1], 2] made extensive use of Cauchy spaces in his work on completions of lattices and lattice ordered groups, and McKennon [17] applied the notion of a Cauchy space in his study of $C^{*}$-algebras. Applications of Cauchy spaces to the study of function spaces can be found in the monograph by Lowen-Colebunders [16].

Kowalsky [13] and Cook and Fischer [5] introduced diagonal axioms for convergence spaces. A convergence structure obeys Cook and Fischer's diagonal axiom if and only if it is topological, whereas, a pretopological convergence structure satisfying Kowalsky's diagonal condition must be topological. Moreover, Cook and Fischer [5] showed that a convergence space is regular if and only if it obeys the "dual" of their diagonal axiom. Kent and Richardson [12] introduced a diagonal condition for Cauchy spaces and proved that a Cauchy space has a topological completion exactly when it satisfies this diagonal axiom. Unfortunately, the "dual" of the above mentioned diagonal condition fails to imply that the Cauchy space completion, or even the underlying Cauchy space, is regular. The dual of the diagonal axiom introduced here is used in characterizing when a strict, regular completion exists. The study of regular completions of Cauchy spaces was initiated by Ramaley and Wyler [19]. Regularity of Kowalsky's completion was characterized by E. Lowen [14].

Received by the editors September 23, 1997 and, in revised form, July 7, 1998.

1991 Mathematics Subject Classification. Primary 54A20, 54D35.

Key words and phrases. Convergence space, Cauchy space, regularity, completion, diagonal axiom.

(C)2000 American Mathematical Society 


\section{Preliminaries}

Let $X$ be a set, $\mathbf{F}(X)$ the set of all (proper) filters on $X$, and $2^{X}$ the set of all subsets of $X$. For $x \in X$, denote by $\dot{x}$ the fixed ultrafilter generated by $\{x\}$. A filter $\mathcal{F}$ is finer (coarser) than a filter $\mathcal{G}$ if $\mathcal{G} \subseteq \mathcal{F}(\mathcal{F} \subseteq \mathcal{G})$ and is denoted by $\mathcal{F} \geq \mathcal{G}(\mathcal{G} \leq \mathcal{F})$. Given $\mathcal{F}, \mathcal{G} \in \mathbf{F}(X)$, the supremum of $\mathcal{F}$ and $\mathcal{G}$, denoted $\mathcal{F} \vee \mathcal{G}$, exists provided $F \cap G \neq \emptyset$ for each $F \in \mathcal{F}, G \in \mathcal{G}$, and is defined to be the smallest filter containing both $\mathcal{F}$ and $\mathcal{G}$.

Definition 2.1. A convergence structure $q$ on a set $X$ is a function $q: \mathbf{F}(X) \rightarrow 2^{X}$ satisfying:

$\left(C_{1}\right) x \in q(\dot{x})$, for all $x \in X$;

$\left(C_{2}\right) \mathcal{F} \leq \mathcal{G}$ implies $q(\mathcal{F}) \subseteq q(\mathcal{G})$

$\left(C_{3}\right) x \in q(\mathcal{F})$ implies $x \in q(\mathcal{F} \cap \dot{x})$.

The pair $(X, q)$ is called a convergence space, and $x \in q(\mathcal{F})$ is interpreted to mean " $\mathcal{F} q$-converges to $x$," and is usually written " $\mathcal{F} \stackrel{q}{\rightarrow} x$." If $q$ is a convergence structure on $X$, then a function $f:(X, q) \rightarrow(Y, p)$ between convergence spaces is continuous provided $f(\mathcal{F}) \stackrel{p}{\rightarrow} f(x)$ whenever $\mathcal{F} \stackrel{q}{\rightarrow} x$. If $p$ and $q$ are convergence structures on $X$ and $f:(X, q) \rightarrow(X, p)$ is continuous, where $f$ is the identity map on $X$, then we write $p \leq q(p$ is coarser than $q$, or $q$ is finer than $p)$.

The closure operator $c l_{q}$ and interior operator $I_{q}$ associated with each convergence space $(X, q)$ are defined for each $A \in 2^{X}$ as follows:

$c_{q} A=\{x \in X: \mathcal{F} \stackrel{q}{\rightarrow} x$ for some $A \in \mathcal{F}\} ;$

$I_{q} A=\{x \in A: \mathcal{F} \stackrel{q}{\rightarrow} x$ implies $A \in \mathcal{F}\}$.

If $\mathcal{F}$ is a filter on $X, c l_{q} \mathcal{F}$ denotes the filter whose base is $\left\{c l_{q} F: F \in \mathcal{F}\right\}$, and a convergence space $(X, q)$ is called regular if $\mathcal{F} \stackrel{q}{\rightarrow} x$ implies $c l_{q} \mathcal{F} \stackrel{q}{\rightarrow} x$.

For every $x \in X$, the $q$-neighborhood filter at $x$ is defined to be

$$
\mathcal{V}_{q}(x)=\left\{V \subseteq X: x \in I_{q} V\right\},
$$

and $A \subseteq X$ is called $q$-open when $I_{q} A=A$. Moreover, $q$ is called a pretopology if $\mathcal{V}_{q}(x) \stackrel{q}{\rightarrow} x$ for each $x \in X$, and a pretopology is a topology if each $\mathcal{V}_{q}(x)$ has a base of $q$-open sets.

Cook and Fischer [5] defined the following two diagonal conditions for convergence spaces in terms of a "compression operator" for filters. These conditions, denoted by $F$ and $F^{\prime}$, are dual to each other in the sense that $F^{\prime}$ may be obtained from $F$ by reversing the implication in the last sentence of $F$, and vice-versa. Let $X$ and $Y$ be non-empty sets, $\mathcal{F} \in \mathbf{F}(Y)$, and $\sigma: Y \rightarrow \mathbf{F}(X)$. Define

$$
K \sigma \mathcal{F}=\bigcup_{F \in \mathcal{F}} \bigcap_{y \in F} \sigma(y)
$$

$K$ is called the "compression operator for $\mathcal{F}$ relative to $\sigma$. " In order to avoid repetition, let us name the following statement.

$\Delta$ : Suppose that $(Z, r)$ is a convergence space containing $(X, q)$ as a subspace. Assume that $\psi: Y \rightarrow Z$ and $\sigma: Y \rightarrow \mathbf{F}(X)$ satisfies $\widetilde{\sigma(y)} \stackrel{r}{\rightarrow} \psi(y)$ for each $y \in Y$, where $\widetilde{\sigma(y)}$ denotes the filter on $Z$ whose basis is $\sigma(y)$. Then the couple $(\psi, \sigma)$ is said to be an exterior selection of $(Z, r)$.

Given a convergence space $(X, q)$, diagonal conditions $F$ and $F^{\prime}$ can be stated in terms of $\Delta$ with $(Z, r)=(X, q)$. 
$F:$ For every exterior selection $(\psi, \sigma)$ of $(X, q)$ and each $\mathcal{F} \in \mathbf{F}(Y), \psi \mathcal{F} \stackrel{q}{\rightarrow} x$ implies that $K \sigma \mathcal{F} \stackrel{q}{\rightarrow} x$.

$F^{\prime}:$ For every exterior selection $(\psi, \sigma)$ of $(X, q)$ and each $\mathcal{F} \in \mathbf{F}(Y), K \sigma \mathcal{F} \stackrel{q}{\rightarrow} x$ implies that $\psi \mathcal{F} \stackrel{q}{\rightarrow} x$.

Important results pertaining to these two axioms are summarized below.

Theorem 2.2. Let $(X, q)$ be a convergence space.

(1) [7] $(X, q)$ is topological if and only if it satisfies $F$.

(2) [5] $(X, q)$ is regular if and only if it satisfies $F^{\prime}$.

Definition 2.3. A Cauchy structure $\mathcal{C}$ on a set $X$ is a collection of filters on $X$ satisfying:

$\left(C H Y_{1}\right) \dot{x} \in \mathcal{C}$, for all $x \in X$;

$\left(C H Y_{2}\right) \mathcal{F} \in \mathcal{C}$ and $\mathcal{F} \leq \mathcal{G}$ implies $\mathcal{G} \in \mathcal{C}$;

$\left(C H Y_{3}\right)$ If $\mathcal{F}, \mathcal{G} \in \mathcal{C}$ and $\mathcal{F} \vee \mathcal{G}$ exists, then $\mathcal{F} \cap \mathcal{G} \in \mathcal{C}$.

A pair $(X, \mathcal{C})$ consisting of a set $X$ and a Cauchy structure $\mathcal{C}$ on $X$ is called a Cauchy space. For each Cauchy space $(X, \mathcal{C})$, there is an associated convergence structure $q_{\mathcal{C}}$ on $X$ defined by $\mathcal{F} \stackrel{q_{\mathcal{C}}}{\rightarrow} x$ iff $\mathcal{F} \cap \dot{x} \in \mathcal{C}$. A Cauchy space $(X, \mathcal{C})$ is $\mathbf{T}_{2}$ (or Hausdorff $)$ if $\left(X, q_{\mathcal{C}}\right)$ is $T_{2}$ in the sense that each $q_{\mathcal{C}}$-convergent filter has a unique limit. A Cauchy space $(X, \mathcal{C})$ is regular if $c l_{q_{\mathcal{C}}} \mathcal{F} \in \mathcal{C}$ whenever $\mathcal{F} \in \mathcal{C}$. It should be mentioned that $\left(X, q_{\mathcal{C}}\right)$ is regular whenever $(X, \mathcal{C})$ is regular, but not conversely. A Cauchy space $(X, \mathcal{C})$ is called complete if every filter $\mathcal{F}$ in $\mathcal{C}$ is $q_{\mathcal{C}}$-convergent. Keller [9] showed that a convergence space $(X, q)$ is induced by some (complete) Cauchy space iff for each distinct pair of points $x, y \in X$, the set of all filters which $q$-converge to $x$ is either equal to or disjoint from the set of all filters which $q$-converge to $y$. Indeed, a sufficient condition occurs when $(X, q)$ is Hausdorff.

Let $(X, \mathcal{C})$ be a Cauchy space. An equivalence relation $\sim$ on $\mathcal{C}$ is defined as follows: For $\mathcal{F}, \mathcal{G} \in \mathcal{C}, \mathcal{F} \sim \mathcal{G}$ iff $\mathcal{F} \cap \mathcal{G} \in \mathcal{C}$. If $\mathcal{F} \in \mathcal{C}$, let $[\mathcal{F}]_{\mathcal{C}}=\{\mathcal{G} \in \mathcal{C}: \mathcal{F} \sim \mathcal{G}\}$ be the equivalence class determined by $\mathcal{F}$; this equivalence class is denoted simply by $[\mathcal{F}]$ if there is no ambiguity. Given Cauchy spaces $(X, \mathcal{C})$ and $(Y, \mathcal{D})$, a map $f:(X, \mathcal{C}) \rightarrow(Y, \mathcal{D})$ is Cauchy continuous if $\mathcal{F} \in \mathcal{C}$ implies $f(\mathcal{F}) \in \mathcal{D}$. If, in addition, $f:(X, \mathcal{C}) \rightarrow(Y, \mathcal{D})$ is a bijection such that $f$ and $f^{-1}$ are both Cauchy continuous, then $f$ is a Cauchy isomorphism. Let $(X, \mathcal{C})$ be a Cauchy space and $A \subseteq X$. A filter $\mathcal{F} \in \mathbf{F}(X)$ has a trace on $A$ if $F \cap A \neq \emptyset$, for each $F \in \mathcal{F}$. In this case, $\mathcal{F}_{A}=\{F \cap A: F \in \mathcal{F}\}$ denotes the trace of $\mathcal{F}$ on $A ; \mathcal{C}_{A}=\left\{\mathcal{F}_{A}: \mathcal{F} \in \mathcal{C}, \mathcal{F}\right.$ has a trace on $A\}$ is a Cauchy structure on $A$, and $\left(A, \mathcal{C}_{A}\right)$ is a Cauchy subspace of $(X, \mathcal{C})$. if

Given a Cauchy space $(X, \mathcal{C})$, the triple $(Y, \mathcal{D}, \theta)$ is called a completion of $(X, \mathcal{C})$

(1) $(Y, \mathcal{D})$ is complete;

(2) $\theta:(X, \mathcal{C}) \rightarrow\left(\theta(X), \mathcal{D}_{\theta(X)}\right)$ is a Cauchy isomorphism;

(3) $c l_{q_{\mathcal{D}}} \theta(X)=Y$.

Moreover, the completion $(Y, \mathcal{D}, \theta)$ is said to be strict if for each $\mathcal{H} \in \mathcal{D}$ there exists $\mathcal{F} \in \mathcal{C}$ such that $c l_{q_{\mathcal{D}}} \theta(\mathcal{F}) \leq \mathcal{H}$.

Several closely related diagonal axioms for a Cauchy space are considered. Let $(X, \mathcal{C})$ be a Cauchy space, $\mathcal{N}$ the set of all $\mathcal{F} \in \mathcal{C}$ which fail to $q_{\mathcal{C}}$-converge, $X^{*}=$ $X \cup\{[\mathcal{F}]: \mathcal{F} \in \mathcal{N}\}$, and define $j: X \rightarrow X^{*}$ by $j(x)=x$ for each $x \in X$. Define $q *$ to be the finest convergence structure on $X^{*}$ such that $j \mathcal{G} \stackrel{q^{*}}{\rightarrow} x$ when $\mathcal{G} \stackrel{q}{\rightarrow} x$ 
and $j \mathcal{F} \stackrel{q^{*}}{\rightarrow}[\mathcal{F}]$ provided $\mathcal{F} \in \mathcal{N}$. The following diagonal axiom for a Cauchy space $(X, \mathcal{C})$ appears in [12].

$D$ : For every exterior selection $(\psi, \sigma)$ of $\left(X^{*}, q^{*}\right)$ and each $\mathcal{L} \in \mathbf{F}(Y), q^{*}$ convergence of $\psi \mathcal{L}$ implies that $K \sigma \mathcal{L} \in \mathcal{C}$.

Observe that if $(\psi, \sigma)$ is an exterior selection of $\left(X^{*}, q^{*}\right)$, then $\sigma(y) \stackrel{q_{\mathcal{C}}}{\rightarrow} \psi(y)$ when $\psi(y) \in X$ and $\sigma(y) \in[\mathcal{F}]$ provided $\psi(y)=[\mathcal{F}]$. The relevance of axiom $D$ is described in the next result.

Theorem 2.4 ([12]). If $(X, \mathcal{C})$ is a Cauchy space, then $(X, \mathcal{C})$ has a topological completion iff it satisfies $D$.

\section{Diagonal COnditions And Regularity}

Let $C H Y$ denote the category whose objects are Cauchy spaces and whose morphisms are Cauchy continuous functions. Using the notation defined in Section 2, a map $\lambda: X^{*} \rightarrow \mathbf{F}(X)$ is called admissible if $\lambda(x)=\dot{x}$ and $\lambda([\mathcal{F}]) \sim \mathcal{F}$ for each $x \in X$ and $\mathcal{F} \in \mathcal{N}$. Let $\Lambda$ denote a nonempty collection of admissible maps, and for $\lambda \in \Lambda, A \subseteq X$, define

$$
A^{\lambda}=A \cup\{[\mathcal{F}]: A \in \lambda[\mathcal{F}]\} .
$$

Observe that $A^{\lambda} \cap B^{\lambda}=(A \cap B)^{\lambda}$ and let $\mathcal{G}^{\lambda}$ be the filter on $X^{*}$ whose base is $\left\{A^{\lambda}: A \in \mathcal{G}\right\}$. Define the convergence structure $p_{\Lambda}$ on $X^{*}$ as follows:

(i) $\mathcal{H} \stackrel{p_{\Lambda}}{\longrightarrow} x$ iff for each $\lambda \in \Lambda$, there exists $\mathcal{F} \stackrel{q_{\mathcal{C}}}{\longrightarrow} x$ such that $\mathcal{H} \geq \mathcal{F}^{\lambda}$,

(ii) $\mathcal{H} \stackrel{p_{\Lambda}}{\longrightarrow}[\mathcal{F}]$ iff for each $\lambda \in \Lambda$, there exists $\mathcal{G} \sim \mathcal{F}$ for which $\mathcal{H} \geq \mathcal{G}^{\lambda}$, and denote $\mathcal{C}^{\Lambda}=\left\{\mathcal{H} \in \mathbf{F}\left(X^{*}\right): \mathcal{H} p_{\Lambda}\right.$-converges $\}$. Reed [20] shows that $\left(X^{*}, \mathcal{C}^{\Lambda}, j\right)$ is a completion of $(X, \mathcal{C})$ in standard form; that is, $j \mathcal{F} \stackrel{p_{\Lambda}}{\longrightarrow}[\mathcal{F}]$ when $\mathcal{F} \in \mathcal{N}$. Reed proved this result in the $T_{2}$ setting, but it is valid without this assumption. When $\Lambda$ consists of all possible admissible maps, $\left(X^{*}, \mathcal{C}^{\Lambda}, j\right)$ becomes Kowalsky's completion 13 and is included in Reed's family of completions. It is of interest to note that the above scheme resembles that used to define the Stone topology on the set of all maximal ideals of $\mathbf{C}^{*}(X)$, where $\mathbf{C}^{*}(X)$ denotes the set of all bounded continuous functions on a completely regular topological space $X$ (e.g., see [8], p. 105).

Given an object $(X, \mathcal{C}) \in|C H Y|, \lambda \in \Lambda$, and subsets $A$ and $B$ of $X$, define $A<^{\lambda} B$ iff $c l_{q_{\mathcal{C}}} A \subseteq B$ and $A \in \mathcal{G} \sim \mathcal{F}$ implies that $B \in \lambda([\mathcal{F}])$. Observe that if $A_{i}<^{\lambda} B_{i}, i=1,2$, then $A_{1} \cap A_{2}<^{\lambda} B_{1} \cap B_{2}$. Moreover, if $\mathcal{F} \in \mathbf{F}(X)$, let $s_{\lambda} \mathcal{F}$ denote the filter on $X$ whose base is $\left\{A \subseteq X: F<^{\lambda} A\right.$ for some $\left.F \in \mathcal{F}\right\}$. These definitions are given by E. Lowen [14] in the $T_{2}$ setting and used to show that Kowalsky's completion is regular iff $\mathcal{F} \in \mathcal{C}$ implies $s_{\lambda} \mathcal{F} \in \mathcal{C}$ for each $\lambda \in \Lambda$. It is shown here that regularity of Reed's family of completions can be characterized using the dual of the following diagonal condition.

$D_{\lambda}:$ Fix $\lambda \in \Lambda$. Then for every exterior selection $(\psi, \sigma)$ of $\left(X^{*}, q^{*}\right)$ and every $\mathcal{L} \in \mathbf{F}(Y), \psi \mathcal{L} \geq \mathcal{F}^{\lambda}$ for some $\mathcal{F} \in \mathcal{C}$, implies that $K \sigma \mathcal{L} \in \mathcal{C}$.

The dual of $D_{\lambda}$, denoted by $D_{\lambda}^{\prime}$, is obtained by reversing the last implication in $D_{\lambda}$; that is, $K \sigma \mathcal{L} \in \mathcal{C}$ implies that $\psi \mathcal{L} \geq \mathcal{F}^{\lambda}$ for some $\mathcal{F} \in \mathcal{C}$. The dual of other diagonal axioms discussed here is defined similarly.

Lemma 3.1. Given $(X, \mathcal{C}) \in|C H Y|$ and $\Lambda$, let $p_{\Lambda}$ denote Reed's convergence structure defined above. If $\mathcal{F} \in \mathbf{F}(X)$, then $c l_{p_{\Lambda}} \mathcal{F}^{\lambda}=c l_{p_{\Lambda}} j \mathcal{F}$ for each $\lambda \in \Lambda$ and, in particular, $\left(X^{*}, \mathcal{C}^{\Lambda}, j\right)$ is a strict completion of $(X, \mathcal{C})$. 
Proof. Assume that $A \subseteq X, \lambda \in \Lambda$ and $[\mathcal{G}] \in \operatorname{cl}_{p_{\Lambda}} A^{\lambda}$. Then there exists $\mathcal{H} \stackrel{p_{\Lambda}}{\longrightarrow}[\mathcal{G}]$ such that $A^{\lambda} \in \mathcal{H}$ and $\mathcal{H} \geq \mathcal{G}_{1}^{\lambda}$ for some $\mathcal{G}_{1} \sim \mathcal{G}$. It follows that $\dot{A}^{\lambda} \vee \mathcal{G}_{1}^{\lambda}$ exists and therefore $\dot{A} \vee \mathcal{G}_{1}$ exists. Let $\mathcal{G}_{2}=\dot{A} \vee \mathcal{G}_{1}$; then $A \in \mathcal{G}_{2}$ and $j \mathcal{G}_{2} \stackrel{p_{\Lambda}}{\longrightarrow}[\mathcal{G}]$, and thus $[\mathcal{G}] \in c l_{p_{\Lambda}} j(A)$. A similar argument holds when $x \in \operatorname{cl}_{p_{\Lambda}} A^{\lambda}$, and it follows that $c l_{p_{\Lambda}} A^{\lambda}=c l_{p_{\Lambda}} j(A)$; hence $c l_{p_{\Lambda}} \mathcal{F}^{\lambda}=c l_{p_{\Lambda}} j \mathcal{F}$. Moreover, if $\mathcal{H} \in \mathcal{C}^{\Lambda}$, then $\mathcal{H} \stackrel{p_{\Lambda}}{\longrightarrow} z$ for some $z \in X^{*}$. If $\lambda \in \Lambda$, then $\mathcal{H} \geq \mathcal{F}^{\lambda}$, and thus $\mathcal{H} \geq c l_{p_{\Lambda}} \mathcal{H} \geq c l_{p_{\Lambda}} \mathcal{F}^{\lambda}=c l_{p_{\Lambda}} j \mathcal{F}$ for some $\mathcal{F} \in \mathcal{C}$. Therefore $\left(X^{*}, \mathcal{C}^{\Lambda}, j\right)$ is a strict completion of $(X, \mathcal{C})$.

Theorem 3.2. Assume that $(X, \mathcal{C}) \in|C H Y|$ and $\Lambda$ is a collection of admissible maps. The following conditions are equivalent:

(a) $\left(X^{*}, \mathcal{C}^{\Lambda}, j\right)$ is a strict, regular completion of $(X, \mathcal{C})$.

(b) $(X, \mathcal{C})$ obeys $D_{\lambda}^{\prime}$ for each $\lambda \in \Lambda$.

(c) $\mathcal{F} \in \mathcal{C}$ implies $s_{\lambda} \mathcal{F} \in \mathcal{C}$ for each $\lambda \in \Lambda$.

Proof. It follows, as in the proof given by E. Lowen (114, Theorem 2.2) for Kowalsky's completion, that (a) and (c) are equivalent. It remains to verify (a) iff (b). Assume that (a) is valid, $\lambda \in \Lambda$ is fixed, condition $\Delta$ holds and $K \sigma \mathcal{L} \in \mathcal{C}$. It suffices to show that $\psi \mathcal{L} \geq \operatorname{cl}_{p_{\Lambda}} j(K \sigma \mathcal{L})$. Indeed, since $\left(X^{*}, \mathcal{C}^{\Lambda}\right)$ is regular, $c l_{p_{\Lambda}} j(K \sigma \mathcal{L}) \stackrel{p_{\Lambda}}{\longrightarrow} z$ for some $z \in X^{*}$, and thus $c_{p_{\Lambda}} j(K \sigma \mathcal{L}) \geq \mathcal{G}^{\lambda}$ for some $\mathcal{G} \in \mathcal{C}$. Fix $A \in K \sigma \mathcal{L}$; then there exists $L \in \mathcal{L}$ such that $A \in \bigcap\{\sigma(y): y \in L\}$. If $y \in L$ and $\psi(y) \in X$, then $\sigma(y) \stackrel{q_{\mathcal{C}}}{\rightarrow} \psi(y)$, and thus $\psi(y) \in c_{p_{\Lambda}} j(A)$. Similarly, if $y \in L$ and $\sigma(y) \in \psi(y)=[\mathcal{G}]$, then $j \sigma(y) \stackrel{p_{\Lambda}}{\longrightarrow}[\mathcal{G}]$, and thus $\psi(y) \in c l_{p_{\Lambda}} j(A)$. It follows that $\psi(L) \subseteq c l_{p_{\Lambda}} j(A)$, and hence $\psi \mathcal{L} \geq c l_{p_{\Lambda}} j(K \sigma \mathcal{L})$. Therefore $D_{\lambda}^{\prime}$ is satisfied.

Conversely, suppose that (b) is satisfied and let $\mathcal{H} \in \mathcal{C}^{\Lambda}$. Assume that $\mathcal{H} \stackrel{p_{\Lambda}}{\longrightarrow}[\mathcal{F}]$; it must be shown that $c_{p_{\Lambda}} \mathcal{H} \stackrel{p_{\Lambda}}{\longrightarrow}[\mathcal{F}]$. Fix $\lambda \in \Lambda$; then there exists $\mathcal{F}_{1} \sim \mathcal{F}$ for which $\mathcal{H} \geq \mathcal{F}_{1}^{\lambda}$. Define $Y=\left\{(\mathcal{G}, x): \mathcal{G} \stackrel{q_{\mathcal{C}}}{\rightarrow} x\right\} \cup\{(\mathcal{L},[\mathcal{K}]): \mathcal{L} \sim \mathcal{K}\}$, and for $F \in \mathcal{F}_{1}$, denote $A_{F}=\{(\mathcal{G}, x) \in Y: F \in \mathcal{G}\} \cup\{(\mathcal{L},[\mathcal{K}]) \in Y: F \in \mathcal{L}\}$, and let $\mathcal{A}$ be the filter on $Y$ whose base is $\left\{A_{F}: F \in \mathcal{F}_{1}\right\}$. Define $\psi(\mathcal{G}, x)=x, \psi(\mathcal{L},[\mathcal{K}])=[\mathcal{K}]$, $\sigma(\mathcal{G}, x)=\mathcal{G}$ and $\sigma(\mathcal{L},[\mathcal{K}])=\mathcal{L}$. Since $F$ belongs to the first component of each member of $A_{F}, \mathcal{F}_{1} \leq K \sigma \mathcal{A}$, and thus $K \sigma \mathcal{A} \in \mathcal{C}$. Then $\psi \mathcal{A}=c l_{p_{\Lambda}} j \mathcal{F}_{1}$, and by Lemma 3.1 and $D_{\lambda}^{\prime}, c l_{p_{\Lambda}} \mathcal{H} \geq c l_{p_{\Lambda}} \mathcal{F}_{1}^{\lambda}=c l_{p_{\Lambda}} j \mathcal{F}_{1}=\psi \mathcal{A} \geq \mathcal{G}^{\lambda}$, for some $\mathcal{G} \in \mathcal{C}$. Moreover, $\mathcal{F}_{1} \geq j^{-1}\left(c l_{p_{\Lambda}} j \mathcal{F}_{1}\right) \geq j^{-1}\left(\mathcal{G}^{\lambda}\right)=\mathcal{G}$, and thus $\mathcal{G} \sim \mathcal{F}$. It follows that $c l_{p_{\Lambda}} \mathcal{H} \stackrel{p_{\Lambda}}{\longrightarrow}[\mathcal{F}]$. A similar argument holds when $\mathcal{H} \stackrel{p_{\Lambda}}{\longrightarrow} j(x)$, and thus $\left(X^{*}, \mathcal{C}^{\Lambda}\right)$ is regular.

Given $(X, \mathcal{C}) \in|C H Y|$ and $A \subseteq X$, define $\Sigma A=j\left(c l_{q_{\mathcal{C}}} A\right) \cup\left\{[\mathcal{G}]: A \in \mathcal{G}_{1}\right.$ for some $\left.\mathcal{G}_{1} \sim \mathcal{G}, \mathcal{G} \in \mathcal{N}\right\}$ and observe that $\Sigma(A \cap B) \subseteq \Sigma A \cap \Sigma B$. If $\mathcal{F} \in \mathbf{F}(X)$, then $\Sigma \mathcal{F}$ denotes the filter on $X^{*}$ with filter base $\{\Sigma F: F \in \mathcal{F}\}$. Define the convergence structure $p_{\Sigma}$ on $X$ as follows:

(i) $\mathcal{H} \stackrel{p_{\Sigma}}{\longrightarrow} x$ iff $\mathcal{H} \geq \Sigma \mathcal{G}$ for some $\mathcal{G} \stackrel{q_{\mathcal{C}}}{\rightarrow} x$,

(ii) $\mathcal{H} \stackrel{p_{\Sigma}}{\longrightarrow}[\mathcal{F}]$ iff $\mathcal{H} \geq \Sigma \mathcal{F}_{1}$ for some $\mathcal{F}_{1} \sim \mathcal{F}, \mathcal{F} \in \mathcal{N}$,

and denote $\mathcal{C}^{*}=\left\{\mathcal{H} \in \mathbf{F}\left(X^{*}\right): \mathcal{H} p_{\Sigma}\right.$-converges $\}$. Unlike $\mathcal{C}^{\Lambda}, \mathcal{C}^{*}$ may fail to obey axiom $\left(\mathrm{CHY}_{3}\right)$ of Definition 2.3. However, it is shown by Kent and Richardson ([10], p. 485$)$ that $\left(X^{*}, \mathcal{C}^{*}, j\right)$ is the only possible candidate for a strict, regular completion of $(X, \mathcal{C})$ which is in standard form. It is straightforward to verify that if $\left(X^{*}, \mathcal{C}^{*}\right) \in$ 
$|C H Y|$, then its induced convergence structure is $p_{\Sigma}$. The diagonal axiom given below is used in characterizing the existence of a strict, regular completion.

Let $A$ and $B$ be subsets of $X$ and define $A<B$ iff (i) $c l_{q_{\mathcal{C}}} A \subseteq B$, and (ii) $\dot{\Sigma A} \vee \Sigma \mathcal{G}$ exists implies $B \in \mathcal{G}_{1}$ for some $\mathcal{G}_{1}$ such that $\mathcal{G}_{1} \stackrel{q_{\mathcal{C}}}{\rightarrow} x$ when $\mathcal{G} \stackrel{q_{\mathcal{C}}}{\rightarrow} x$, or $\mathcal{G}_{1} \sim \mathcal{G}$ if $\mathcal{G} \in \mathcal{N}$. If $\mathcal{F} \in \mathbf{F}(X)$, denote $t \mathcal{F}$ to be the filter on $X$ whose subbase is $\{A \subseteq X: F<A$ for some $F \in \mathcal{F}\}$, and observe that $t \mathcal{F} \leq c l_{q_{\mathcal{C}}} \mathcal{F}$. Moreover, $(X, \mathcal{C})$ is said to be $t$-closed when $\mathcal{F} \in \mathcal{C}$ implies $t \mathcal{F} \in \mathcal{C}$. Consider the following diagonal axiom for $(X, \mathcal{C})$.

$D_{\Sigma}$ : Given any exterior selection $(\psi, \sigma)$ of $\left(X^{*}, q^{*}\right)$ and $\mathcal{L} \in \mathbf{F}(Y), \psi \mathcal{L} \geq \Sigma \mathcal{G}$ for some $\mathcal{G} \in \mathcal{C}$, implies that $K \sigma \mathcal{L} \in \mathcal{C}$.

Theorem 3.3. The Cauchy space $(X, \mathcal{C})$ has a strict, regular completion iff all three of the following conditions are satisfied:

(a) $\Sigma \mathcal{F} \vee \Sigma \mathcal{G}$ exists implies $\mathcal{F} \cap \mathcal{G} \in \mathcal{C}$, when $\mathcal{F}, \mathcal{G} \in \mathcal{C}$.

(b) $(X, \mathcal{C})$ is t-closed.

(c) $(X, \mathcal{C})$ obeys $D_{\Sigma}^{\prime}$.

Proof. Assume that $(X, \mathcal{C})$ has a strict, regular completion. Then $\left(X^{*}, \mathcal{C}^{*}, j\right)$ is the only one in standard form and thus (a) must be valid. It is shown that $(X, \mathcal{C})$ is $t$ closed. Indeed, if $\mathcal{F} \in \mathcal{C}$, then $c l_{p_{\Sigma}} \Sigma \mathcal{F} \geq \Sigma \mathcal{G}$ for some $\mathcal{G} \in \mathcal{C}$, and it suffices to show that $c l_{q_{\mathcal{C}}} \mathcal{G} \leq t \mathcal{F}$. Fix $G \in \mathcal{G}$; then there exists $F \in \mathcal{F}$ for which $c l_{p_{\Sigma}} \Sigma F \subseteq \Sigma G$, and it remains to verify that $F<c_{q_{\mathcal{C}}} G$. Suppose that $\Sigma F \vee \Sigma \mathcal{K}$ exists for $\mathcal{K} \in \mathcal{N}$. Then $[\mathcal{K}] \in c l_{p_{\Sigma}} \Sigma F \subseteq \Sigma G=c l_{p_{\Sigma}} j G$, and thus there exists $\mathcal{H} \stackrel{p_{\Sigma}}{\longrightarrow}[\mathcal{K}]$ such that $j(G) \in \mathcal{H}$ and $\mathcal{H} \geq \Sigma \mathcal{K}_{1}$ for some $\mathcal{K}_{1} \sim \mathcal{K}$. Since $j(G) \in \mathcal{H}, G \in j^{-1} \mathcal{H} \geq j^{-1}\left(\Sigma \mathcal{K}_{1}\right)=$ $c l_{q_{\mathcal{C}}} \mathcal{K}_{1} \sim \mathcal{K}$, because $(X, \mathcal{C})$ is regular; hence $G \in j^{-1} \mathcal{H} \sim \mathcal{K}$. A similar argument

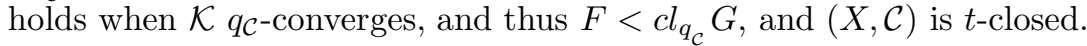

Next, it is shown that $D_{\Sigma}^{\prime}$ is valid. Assume that condition $\Delta$ is satisfied and $K \sigma \mathcal{L} \in \mathcal{C}$. An argument similar to the one given in the first part of Theorem 3.2 shows that $\psi \mathcal{L} \geq c l_{p_{\Sigma}} j(K \sigma \mathcal{L})$, and since $\left(X^{*}, \mathcal{C}^{*}\right)$ is regular, $\psi \mathcal{L} \geq \Sigma \mathcal{G}$ for some $\mathcal{G} \in \mathcal{C}$. Hence $D_{\Sigma}^{\prime}$ is satisfied.

Conversely, assume that (a), (b) and (c) are valid and $\mathcal{F} \in \mathcal{C}$; condition (a) implies that $\left(X^{*}, \mathcal{C}^{*}\right) \in|C H Y|$. It remains to show that $c l_{p_{\Sigma}} \Sigma \mathcal{F} \in \mathcal{C}^{*}$. Define $Y=\left\{(\mathcal{G}, x): \mathcal{G} \stackrel{q_{\mathcal{C}}}{\rightarrow} x\right\} \cup\{(\mathcal{K},[\mathcal{L}]): \mathcal{K} \sim \mathcal{L}, \mathcal{L} \in \mathcal{N}\}, \psi(\mathcal{G}, x)=x, \psi(\mathcal{K},[\mathcal{L}])=[\mathcal{L}]$, $\sigma(\mathcal{G}, x)=\mathcal{G}$ and $\sigma(\mathcal{K},[\mathcal{L}])=\mathcal{K}$. Then $\psi$ and $\sigma$ satisfy the conditions of $\Delta$. Suppose that $F \in \mathcal{F}$ and $F<A$; define $L_{F, A}=\left\{(\mathcal{G}, x) \in Y: A \in \mathcal{G}\right.$ and $\dot{\Sigma} F \vee \Sigma \mathcal{G}_{1}$ exists for some $\left.\mathcal{G}_{1} \stackrel{q_{\mathcal{C}}}{\rightarrow} x\right\} \cup\left\{(\mathcal{K},[\mathcal{L}]) \in Y: A \in \mathcal{K}\right.$ and $\Sigma F \vee \Sigma \mathcal{K}_{1}$ exists for some $\left.\mathcal{K}_{1} \sim \mathcal{L}\right\}$. Observe that if $F_{i}<A_{i}, F_{i} \in \mathcal{F}$, for $i=1,2$, and $x \in F_{1} \cap F_{2}$, then $(\dot{x}, x) \in L_{F_{1}, A_{1}} \cap L_{F_{2}, A_{2}}$ and thus the intersection is nonempty. Let $\mathcal{L}$ denote the filter on $Y$ whose subbase is $\left\{L_{F, A}: F<A, F \in \mathcal{F}\right\}$. Fix $F<A, F \in \mathcal{F}$ and note that $\psi\left(L_{F, A}\right) \subseteq c l_{p_{\Sigma}} \Sigma F$. Conversely, assume that $x \in c l_{p_{\Sigma}} \Sigma F$; then $\dot{\Sigma} F \vee \mathcal{G}_{1}$ exists for some $\mathcal{G}_{1} \stackrel{q_{\mathcal{C}}}{\rightarrow} x$. Since $F<A, A \in \mathcal{G}$ for some $\mathcal{G} \stackrel{q_{\mathcal{C}}}{\rightarrow} x$, and thus $(\mathcal{G}, x) \in L_{F, A}$ and $x \in \psi\left(L_{F, A}\right)$. A similar argument is valid when $[\mathcal{L}] \in \operatorname{cl}_{p_{\Sigma}} \Sigma F$, and thus $\psi\left(L_{F, A}\right)=c l_{p_{\Sigma}} \Sigma F \in c l_{p_{\Sigma}} \Sigma \mathcal{F}$. It follows that $\psi \mathcal{L} \leq c l_{p_{\Sigma}} \Sigma \mathcal{F}$.

Finally, it is shown that $t \mathcal{F} \leq K \sigma \mathcal{L}$. If $A$ is a member of the subbase for $t \mathcal{F}$, then $F<A$ for some $F \in \mathcal{F}$. Observe that $A \in \bigcap\left\{\sigma(z): z \in L_{F, A}\right\}$, and thus $t \mathcal{F} \leq K \sigma \mathcal{L}$. Since $(X, \mathcal{C})$ is $t$-closed, $K \sigma \mathcal{L} \in \mathcal{C}$, and hence by $D_{\Sigma}^{\prime}, \psi \mathcal{L} \in \mathcal{C}^{*}$. However, $\psi \mathcal{L} \leq c l_{p_{\Sigma}} \Sigma \mathcal{F}$, and thus $c l_{p_{\Sigma}} \Sigma \mathcal{F} \in \mathcal{C}^{*}$. Therefore $\left(X^{*}, \mathcal{C}^{*}\right)$ is regular. 


\section{Concluding Remarks}

Several papers have been written concerning notions of completeness in spaces which are closely related to Cauchy spaces. Recent work in this direction can be found in Csaszar [6] and references therein. It would be of interest to equip a probabilistic convergence space (see [21]) with a notion of completeness. The latter concept originated with Menger [18, who proposed replacing the numerical distance between two points with a probability distribution function in order to account for inherent uncertainties of measurements in physical situations. Subsequent work in this area appears in the book by Schweizer and Sklar [22.

Diagonal axioms continue to be a source of interest. Recent work in this area includes [3, [15] and [23. A current paper relating diagonal conditions and convergence notions is due to Wyler [24].

\section{ACKNowledgement}

The authors are grateful to the referee for making suggestions that led to a significant improvement of the presentation.

\section{REFERENCES}

[1] R. Ball, "Convergence and Cauchy Structures on Lattice Ordered Groups," Trans. Amer. Math. Soc. 259 (1980), 357-392. MR 81m:06039

[2] R. Ball, "Distributive Cauchy Lattices," Algebra Universalis 18 (1984), 134-174. MR 86a:06015

[3] P. Brock and D.C. Kent, "Approach Spaces, Limit Tower Spaces, and Probabilistic Convergence Spaces," Applied Categorical Structures 5 (1997) 439-452. MR 98b:54017

[4] C. H. Cook and H. R. Fischer, "Uniform Convergence Structures," Math. Ann. 173 (1967), 290-306. MR 36:845

[5] C. H. Cook and H. R. Fischer, "Regular Convergence Spaces", Math. Ann. 174 (1967), 1-7. MR 37:5837

[6] A. Csaszar, " $\lambda$-Complete Filter Spaces," Acta Math. Hungar. 70(1996), 75-87. MR 97d:54005

[7] S. Dolecki and G. H. Greco, "Cyrtologies of Convergences," Math. Nachr. 126 (1986), 327-348. MR 88b:54002a

[8] L. Gillman and M. Jerison, Rings of Continuous Functions, Springer-Verlag, Graduate Texts in Mathematics (1976). MR 53:11352

[9] H. Keller, " Die Limesuniformisierbarkeit der Limesräume," Math. Ann. 176 (1968), 334-341 MR 37:874

[10] D. Kent and G. Richardson, "Regular Completions of Cauchy Spaces," Pac. J. Math. 51 (1974), 483-490. MR 52:11811

[11] D. Kent and G. Richardson, "Cauchy Spaces with Regular Completions," Pac. J. Math. 111 (1984), 105-116. MR 85f:54007.

[12] D. Kent and G. Richardson, "Diagonal Cauchy Spaces," Bull. Austral. Math. Soc. 54(1996), 255-265. MR 98h:54037

[13] H. J. Kowalsky, "Limesräume und Komplettierung," Math. Nachr. 12 (1954), 301-340. MR 17:390b

[14] E. Lowen, "On the Regularity of the Kowalsky Completion," Can. J. Math. 36 (1984), 58-70.

[15] E. Lowen and R. Lowen, "A Quasitopos Containing CONV and $M E T$ as Full Subctegories," Intl. J. Math. and Math. Sci. II (1988), 417-438. MR 89g:54024

[16] E. Lowen-Colebunders, Function Classes of Cauchy Continuous Maps, Marcel Dekker, New York (1989). MR 91c:54002

[17] K. McKennon, "The Strict Topology and the Cauchy Structure of the Spectrum of a $C^{*}$ Algebra," Topology and its Applications 5 (1975), 249-262. MR 52:1333

[18] K. Menger, Statistical Metrics, Proc. Nat. Acad. Sci. USA 28 (1942), 535-537. MR 4:163e

[19] J.F. Ramalay and O. Wyler, Cauchy Spaces II: Regular Completions and Compactifications, Math. Ann. 187 (1970), 187-199. 
[20] E.E. Reed, "Completions of Uniform Convergence Spaces," Math. Ann. 194 (1971), 83-108. MR 45:1109

[21] G.D. Richardson and D.C. Kent, "Probabilistic Convergence Spaces," J. Austral. Math. Soc. (Series A) 61 (1996), 400-420. MR 97j:54031

[22] B. Schweizer and A. Sklar, Probabilistic Metric Spaces, North-Holland, New York (1983). MR 86g:54045

[23] S. A. Wilde, Study and Characterization of $p$-Topological Convergence Spaces, Ph.D. Thesis, Washington State University, 1997.

[24] O. Wyler, "Convergence Axioms for Topology," To Appear.

Department of Mathematics, San Diego State University, San Diego, California 92182-7720

E-mail address: pwbrock@pacbell.net

Department of Mathematics, University of Central Florida, Orlando, Florida 32816 6284

E-mail address: garyr@pegasus.cc.ucf.edu 